\title{
Supporting conceptualisation processes in collaborative networks: a case study on an R\&D project
}

\author{
Carla Pereira, Cristovão Sousa and António Lucas Soares
}

\begin{abstract}
The development of new products or processes involves the creation, re-creation and integration of conceptual models from the related scientific and technical domains. Particularly, in the context of collaborative networks of organisations (CNO) (e.g. a multi-partner, international project) such developments can be seriously hindered by conceptual misunderstandings and misalignments, resulting from participants with different backgrounds or organisational cultures, for example. The research described in this article addresses this problem by proposing a method and the tools to support the collaborative development of shared conceptualisations in the context of a collaborative network of organisations. The theoretical model is based on a socio-semantic perspective, while the method is inspired by the conceptual integration theory from the cognitive semantics field. The modelling environment is built upon a semantic wiki platform. The majority of the article is devoted to developing an informal ontology in the context of a European R\&D project, studied using action research. The case study results validated the logical structure of the method and showed the utility of the method.
\end{abstract}

\section{Keywords}

conceptualisation processes; ontology engineering; collaborative networks

\section{Introduction}

Research and development projects and innovation (RDI) projects in general frequently involve partners from several countries that possess complementary areas of specialisation but apply distinct professional expertise and exhibit different cultures and varying world-views. Even though an RDI project is a human activity system which is domain and time-bound, the above-mentioned characteristics of the partnership pose non-trivial difficulties in exchanging meanings naturally and seamlessly. This process is fundamental to achieve a common conceptualisation of the working domains (scientific, technological, professional, ethical, etc.) involved in the project. As it will be argued in this article, a common conceptualisation of the domains involved in a project is the cornerstone for an effective and efficient management of the organisation of the project (i) information, classification and retrieval, (ii) knowledge sharing and (iii) collaboration governance and support. These three aspects will be called the components of a project's knowledge organisation and collaboration system (KOCS).

Setting up a KOCS in an RDI project implies that the partners share (even if implicitly) a set of conceptual structures (concepts, their descriptions and their relationships) with which the domain or domains of work and the project plan and division of work are to be understood. This usually requires the implicit negotiation of these conceptual structures when constructing and agreeing on a project glossary (potentially in the form of a simplified ontology), an information architecture - document organisation, web pages structure -and the governance schemes of the interaction of the joint activities - which include a semantic agreement. From our experience in participating in several RDI projects, mostly in the area of new manufacturing processes with multiple partners from several countries, the authors have observed that in every project there are numerous problematic situations due to conceptual misalignments between the partners collaborating on a task. Even if the abovementioned process of implicit conceptual negotiation occurs, it is not comprehensive enough to achieve a common conceptualisation in order to achieve an effective KOCS. Surprisingly, as far as the authors are aware, there is no research that addresses or even formulates this problem.

A way to address the problem of conceptual misalignment in a multi-partner project, as described above, is to implement a process of achieving an explicit representation of a common conceptualisation. This ought to be a collaborative process as some kind of negotiation is needed to achieve a common goal: 
every partner must be aligned by the same conceptual reference. However, this way has a serious drawback as it involves strong interaction between the people involved and it is heavily mediated by representational artefacts and it is complex and time consuming. Therefore, it was envisaged another line of research to create tools to support the conceptual negotiation processes.

One possible area to look for research results and commercial tools that can be part of a solution is the Ontology Engineering area. Ontology engineering deals with the development of human understandable, machine processable ontologies, addressing topics such as the ontology development life-cycle, development and evolution methodologies and ontology reuse and integration. In the last couple of years, research on the applications of ontologies in manufacturing and industry in general has been consistently reported as the semantic web (SW) movement continued to grow. Some of this research has addressed ontology development, with more or less detail but until now none of the research has addressed the collaborative development of an ontology for industrial or manufacturing applications and all the surrounding issues. Even in the computer science and artificial intelligence fields, topics such as conceptualisation processes (CPs), conceptual negotiation, shared conceptualisation, conceptual learning and development and ontology specification have not received much attention.

The need for the collaborative development of semantic artefacts in distributed manufacturing or manufacturing networks equates with the needs for the use of ontologies and can be found in several areas. A review of recent applied research on the use and engineering of ontologies for manufacturing and industry in general is presented in Section 2. Even though the most used definition of ontology (Gruber 1993) 'An ontology is a formal, explicit specification of a shared conceptualisation', underlines the collaborative construction of conceptualisations in a scientific context, the reality is quite different: 'While different degrees of formalizations have been well investigated and are now found in various ontology-based technologies, the notion of a shared conceptualisation is neither well-explored, norwell-understood, nor well-supported by most ontology engineering tools' (Staab 2008). Our view is that ontology engineering requires a 'sociocognitive turn' in order to generate tools that are really effective in copying the complex, unstructured and situational contexts that characterise information and knowledge management in businesses collaboration.

As presented in Section 3, our research strategy led us to the application of results from the cognitive semantic field in the creation of artefacts acting as socio-technical devices. This supports the view that meaning is socially constructed through collaboration and negotiation. The first line of this research deals with the application and extension of the conceptual blending theory (CBT) (Fauconnier and Turner 1998) to the realm of collaborative semantic tools. The practical application of our approach is to support the co-construction of semantic artefacts using groups of social actors placed in organisational contexts, interacting and working towards a set of common objectives. A new method to support the collaborative specification of semantic artefacts (such as classification schemes or ontologies) is proposed, with special focus on the initial phase of the process; the conceptualisation phase.

The article begins by describing the conceptual misalignment problem in a concrete setting - multipartner, transnational projects - and details a case involving this type of project. Furthermore, the author's research on collaborative CPs is directed more broadly at collaborative networks of organisations (CNO). The type of projects studied in this article can be framed under the CNO concept in general (Camarinha-Matos and Afsarmanesh 2005) and virtual organisation reference processes (formation, operation and evaluation) in particular (CamarinhaMatos and Afsarmanesh 2007).

The rest of the article is structured as follows: the next section reviews the scientific literature on the development of ontologies for manufacturing applications, including more general approaches that include collaborative processes. Section 3 describes the theoretical and conceptual framework for the integrated modelling of social and semantic networks and outlines the ColBlend method. This is followed by a brief description of the CBT that inspired this approach. The collaborative platform that supports the method is also described briefly. In Section 4, the development of an informal ontology is described in the context of a European project as the setting to validate and improve the ColBlend method. The lessons learnt and recommendations conclude this section and conclusions and future work are presented at the end of this article.

\section{Development of ontologies for manufacturing applications: a review}

\subsection{Definitions}

There are some terms that have been used so far that can be understood differently according to the context in which they are used. Therefore, working definitions for the most relevant terms will now be given.

Starting with the 'specification of a conceptualisation' as the minimal definition of ontology (Gruber 1993), this term is used here with two related meanings: 
(i) to refer to the computational semantic artefact codifying a 'class-relationship-instance' model of the word and (ii) a representation of a conceptualisation through a map of concepts, that equates 'specification' with 'representation'. Sometimes the term 'conceptual representation' or 'representation of a conceptualisation' is used with the same meaning as (ii).

As for the concept of 'conceptualisation', the intuitive definition 'the relevant informal knowledge one can extract and generalize from experience, observation, or introspection' (Prévot 2010) is adopted as a starting point. Continuing in an informal tone, it can be said that a conceptualisation is the result of a ' $\mathrm{CP}$ ' that leads to the extraction and generalisation of relevant information from experience (Pre'vot 2010). For an individual, the $\mathrm{CP}$ of a given reality is a collection of ordered cognitive activities with information and knowledge that is internally or externally accessible to the individual as inputs and an internal or external conceptual representation as the output. Furthermore, a 'collaborative conceptualisation process' (CCP) is a CP that involves more than one individual producing an agreed conceptual representation. In addition to an individual $\mathrm{CP}$, the CCP involves social activities that include the negotiation of meaning and practical management activities for the collaborative process.

The terms 'conceptual structure' and 'knowledge structure' are also used in an informal way as synonyms to refer to extracts of a domain conceptualisation for example, 'supplier requirements', 'supplier performance management' or 'manufacturing process capability'.

\subsection{Ontology engineering for manufacturing applications}

Ontologies and ontology engineering are the cornerstones of the SW which is deemed more and more important for industry and in particular for manufacturing. Breslin et al. (2010) describe the benefits of SW in industry in three main dimensions: knowledge management, enterprise application integration and ecommerce. Applications ranging from personal and corporate information management to data integration in collaborative environments (including design, supply, production and recycling processes) can clearly improve almost every area of manufacturing management. Riss et al. (2010) also foresee that semanticbased systems will play a central role in industry and in manufacturing companies in particular. Communication and on work coordination are among the main organisational dimensions that will suffer big improvements through the adoption of semantic-based systems and technologies. Once again, ontologies (e.g. task and collaboration ontologies) are considered the cornerstone for these approaches.

Rajpathak and Chougule (2011) propose a generic and comprehensive methodology that 'puts ontology engineering on a firm scientific foundation and at the same time provides a collaborative environment for effective knowledge sharing and reuse'. The authors claim that the approach also provides a way of automatically extracting frequent terms from the data to construct an ontology in a bottom-up fashion. A pre-development phase is proposed, including knowledge acquisition tasks such as the extraction of domain-specific concepts from textual documents in a (semi-) automatic fashion to overcome the limitations associated with manual ontology creation and the conduction of (semi-) structured interviews with small and medium enterprises. This approach is based on well-accepted classic methodologies in ontology engineeringbutin ouropinion itinherits their main problem: it considers the achievement of shared conceptualisation to be unproblematic.

Lin et al. (2011) present a constructive, two-level knowledge modelling approach to systematically develop manufacturing ontologies for distributed manufacturing environments using both software engineering and SW paradigms. Although they claim that the approach addresses the improvement of the communication between domain experts and knowledge engineers, their proposal is to use UML/OCL-based models for this purpose and nothing is explained about how the stakeholders negotiate and conceptualise collaboratively to elaborate these models. Once again, it is implicit that a common understanding of the domains that will be represented by the ontology is achieved without any hindrance.

Chungoora et al. (2010) analyses the relative strengths and weaknesses in employing lightweight or full versions of ontologies to represent and share knowledge between multiple domains in injection moulding design and manufacture. They concluded that the process of ontology development should be accompanied by the provision of appropriate ontology design schematics and they propose the unified modelling language (UML) as the tool to represent the ontology specification. Although they present a case study involving the development and use of a multi-domain ontology, no details were given on the process of achieving the ontology specification (UML models) and the adequacy of the UML as a representational tool was not assessed.

\subsection{Generic ontology development methods with collaboration components}

The work on ontology development methodologies has been extensively discussed and compared by Lo'pez 
et al. (1999), Ferna'ndez-Lo'pez and Go'mez-Pérez (2002), Corcho et al. (2003) and Gasevic et al. (2006). By analysing the state-of-the-art, it can be concluded that the ontology engineering field has put a lot of emphasis on the 'specification of the conceptualisation' as an engineering task, giving little importance to the early phases of the ontology development life-cycle. In particular, the importance of the social processes involved in the formation of a shared conceptualisation (e.g. of a domain) has not been recognised at all, although some research has addressed the collaborative dimension of ontology building (see Staab et al. 2001, Sure 2002, Go'mez-Gauchía et al. 2004a, 2004b, Pinto et al. 2004, de Moor et al. 2006; for a complete account of those approaches). From these, the "Knowledge Mediation Procedure" (Aschoff 2004), Application Knowledge Engineering Methodology (AKEM) (Zhao 2005) and Human-Centered Ontology Engineering Methodology (HCOME) (Kotis and Vouros 2006) are the works that address the collaboration dimension as fundamental and develop methods and techniques that can result in some form of shared conceptualisation. Nevertheless, from this literature review, it can be concluded that the importance of supporting the collective construction of a conceptualisation is not recognised. Other particular questions result from this review: (i) the importance of representational tools and user interfaces for interacting with knowledge representations is generally underestimated; (ii) negotiation and consensus building for conceptualisation content has not been a priority either; there are a

few proposals that claim to support the process of reaching a consensus or agreements and only one addresses the issue of what conceptual content should be included in the shared conceptualisation and (iii) the reutilisation of existing ontologies is an obvious

requirement; nevertheless, there is no approach that integrates reutilisation with the $\mathrm{CP}$ in a systematic way.

The methodology described in this article and the tools developed to support it aim to fill this gap in ontology engineering in general and in ontology specification for manufacturing applications in particular.

\section{Supporting the collaborative conceptualisation process}

\subsection{Theoretical framework}

In the line of research presented in this article, the interplay between the social network and the semantic network within a collaborative network of organisations is studied. Therefore, a modelling framework was needed that was able to model (i) social actors relationships, (ii) conceptual structure relationships and (iii) the relationships between (i) and (ii). Broadly, this integrated view would help the CNO researchers understand the interplay between knowledge structures and social structures better. To address this need and depart from the work of Roth (2006) on scientific communities, a conceptual and formal framework was developed, adapting her approach to model organisational networks as socio-semantic networks. Instead of only considering social networks of individuals, a layer was added to model a social network of organisations. Furthermore, instead of only considering single concepts as Roth did, a new layer was added to organise concepts and conceptual structures in business domains (D). The goal was to achieve a framework with adequate expressiveness to model the relationships between a network of organisational actors and the conceptual structures socially constructed/used by them for various purposes. With a set of actors (e.g. individuals and groups in an inter-organisational project team) and a set of domains decomposed in a set of concept structures (e.g. an engineering or management domain, a professional body of knowledge, a market segment discourse, etc., together with their components), a socio-semantic network is a network of relationships between the organisational actors and the concept structures. This relationship can be represented as a bipartite graph with edges that link actors to concepts (see Figure 1). A social network is a set of individuals (nodes), p, of the organisations (partners), s, that compose the collaborative network and a set of edges representing the relationships between the individuals. In a semantic network, I, the nodes are concepts of the various domains involved in the network and the edges are the relationships between the concepts. Consequently, I1 will be the semantic network that represents the conceptual model of s1, I2 represents the conceptual model of $s 2$ and so on. An epistemic network is the composition of the three networks: the social network (involving links between individuals), the semantic network (with links between concepts) and the socio-semantic network (which links individuals to concepts).

Unlike Roth (2006) who only considered one kind of link between a single actor and a single concept, this work considered different kinds of links that can influence the result of the shared CP. In the social network, two kinds of relations are considered: links between the various organisations that constitute the collaborative network (i.e. between individuals from different organisations, these links have an influence during the negotiation process) and links between pairs of individuals within the organisation (these links influence the creation of the conceptualisation proposals). In the semantic network, two kinds of relations are considered: links between concepts of different conceptualisation proposals and links between concepts within each conceptualisation proposal. In the 


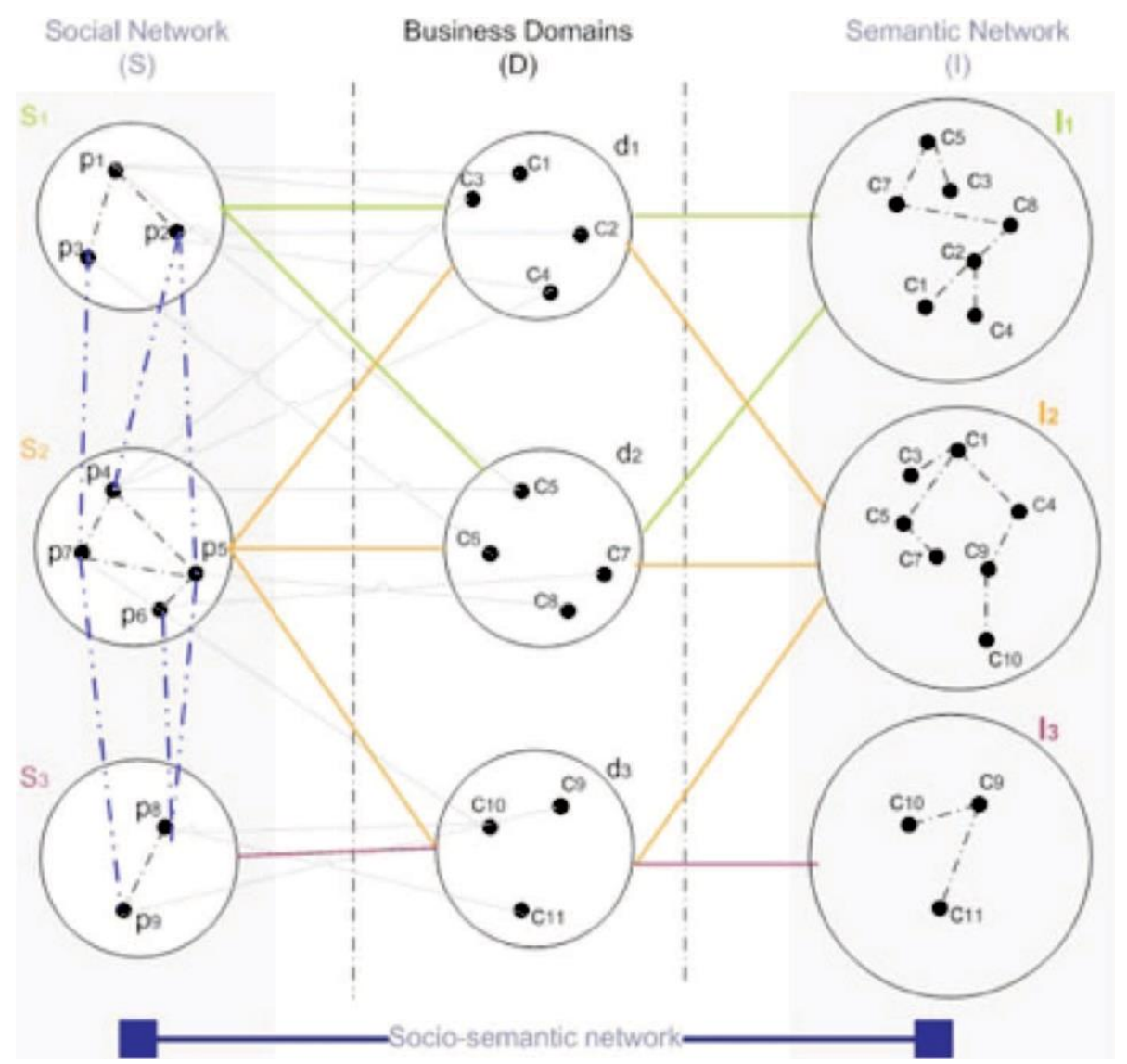

Figure 1. The socio-semantic network model.

socio-semantic network, the links between the individuals that compose the network and the concepts that compose the shared conceptualisation are also considered.

\subsection{The conceptual blending theory}

The proposed method and tool to support the collaborative conceptualisation was based on the CBT from Fauconnier and Turner $(1998,2002)$. The CBT has its roots in the cognitive semantics discipline which advocates that meaning is constructed on a conceptual level (Evans and Green 2006): 'meaning construction is equated with conceptualisation, a dynamic process where linguistic units serve as prompts for an array of conceptual operations and the recruitment of background knowledge'. The CBT accounts for the emergence of meanings by adopting the view that the construction of meaning involves emergent structures, this means that conceptual integration is more than the sum of its component parts. An integration network is thus a mechanism for modelling how emergent meaning might come about, accounting for the dynamic aspects of the construction of meaning. The CBT representation (see Figure 2) gives rise to complex networks by linking two (or more) input spaces by means of a generic space. The generic space provides information that is abstract enough to be common to all of the input spaces. The elements in the generic space are mapped onto counterparts in each of the input spaces, which motivate the identification of cross-space counterparts in the input spaces. A further space in this model of integration network is the blended space or blend. This is the space that contains new or emergent structures: concepts and relations that are not contained in either of the inputs. The blend takes elements from both inputs but goes further and provides additional structures that distinguish the blend from either of its inputs. In other words, the blend can derive structures that are not contained in either input. Anticipating the description of the method in 3.2, the three types of spaces defined in the CBT are mapped in our method spaces in the following way: The input spaces (1) are considered 'conceptualisation proposal spaces' where each organisation participant in the network presents its proposals; the generic space is considered a 'Shared Space' that works as a space where only concepts and 


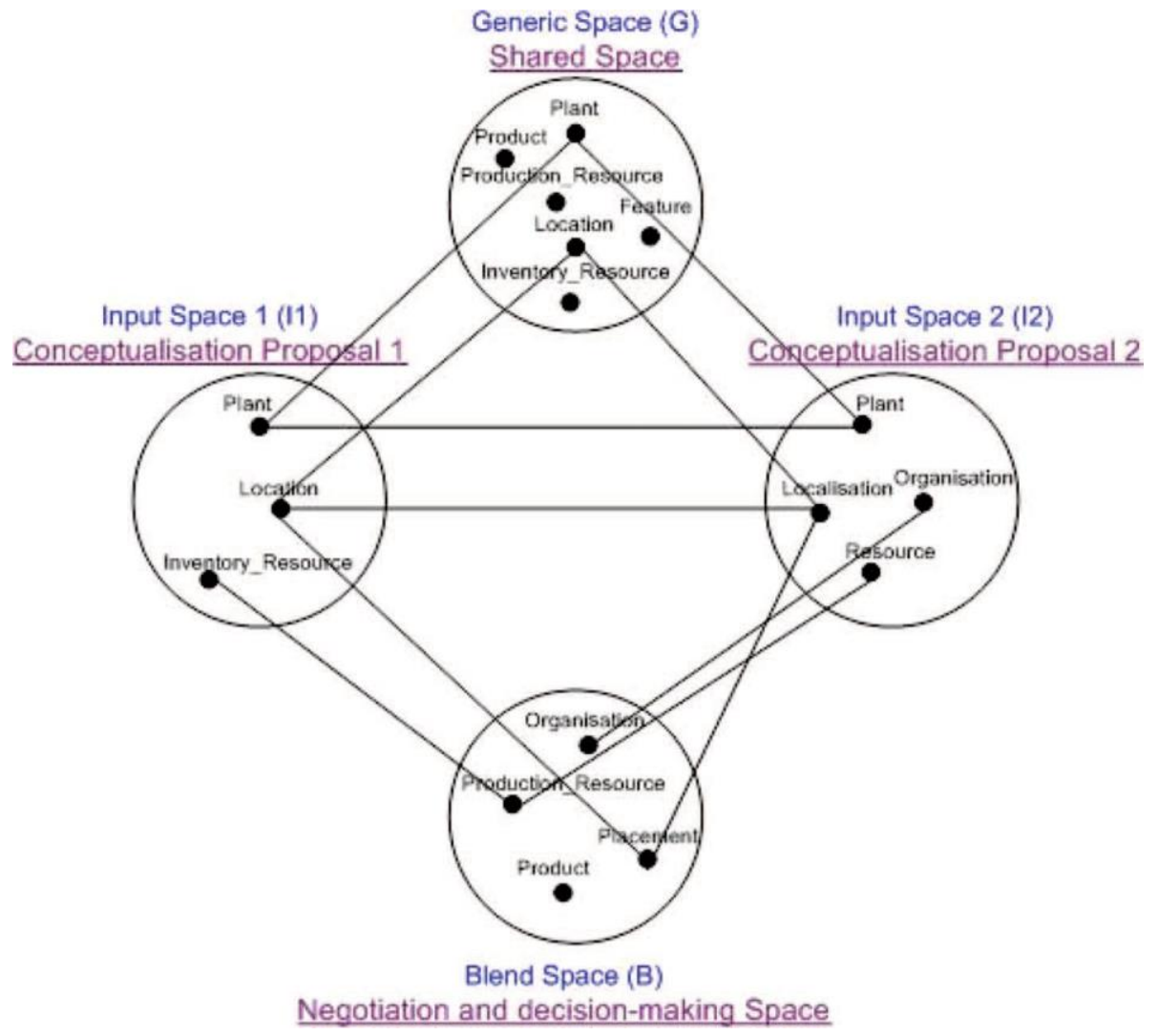

Figure 2. The network model of Conceptual Blending.

relations consensually accepted by all of the actors are represented. The blend space will be considered the 'Negotiation Space' and is used during the conceptual negotiation process.

In the CBT, there are three processes that produce an emergent conceptual structure (Fauconnier and Turner 1998): composition; completion and elaboration. The first process involves the composition of elements from separate input spaces.

In the example above, the composition brings together the concept, INVENTORY_RESOURCE, of input space 1 with the concept, RESOURCE, of input space 2 resulting in PRODUCTION_RESOURCE in the blend. Similarly, PLACEMENT in the blend composes the elements projected from the input space 1, LOCATION, with those projected from the input space 2, LOCALISATION. The second process, completion, involves the introduction of schema. This involves the unconscious and effortless recruitment of background frames and these complete the composition. Without the structure provided by the context frame, it would lose the central inference emerging from the blend. This process of schema introduction is called 'completion' because the structure is recruited to 'fill out' or complete the information projected from the inputs in order to derive the blend. Finally, elaboration is the process that produces the structure that is unique to the blend. This process is also called running the blend. For example, the concept PRODUCT in the blend results from this process. The CBT has more to say about these constitutive processes but further description is not within the scope of this article (see, e.g. Fauconnier and Turner 1998). In summary, the CBT can be used as an instrument to understand, negotiate and agree on common conceptual structures of a domain, leading to 'alignment' between the network social structure and the semantic networks linked to each actor (see Figure 3).

\subsection{The ColBlend method}

\subsubsection{Steps for the method}

Based on the theory outlined in the previous sections, a method and tool were developed to support collaborative CPs. The goal was then to achieve an agreed set of conceptual models, through a process involving explanation, discussion and negotiation. The preconditions for the execution of this method are: (i) a collaborative network that has been formed and its goals and mission are defined and understood by all 


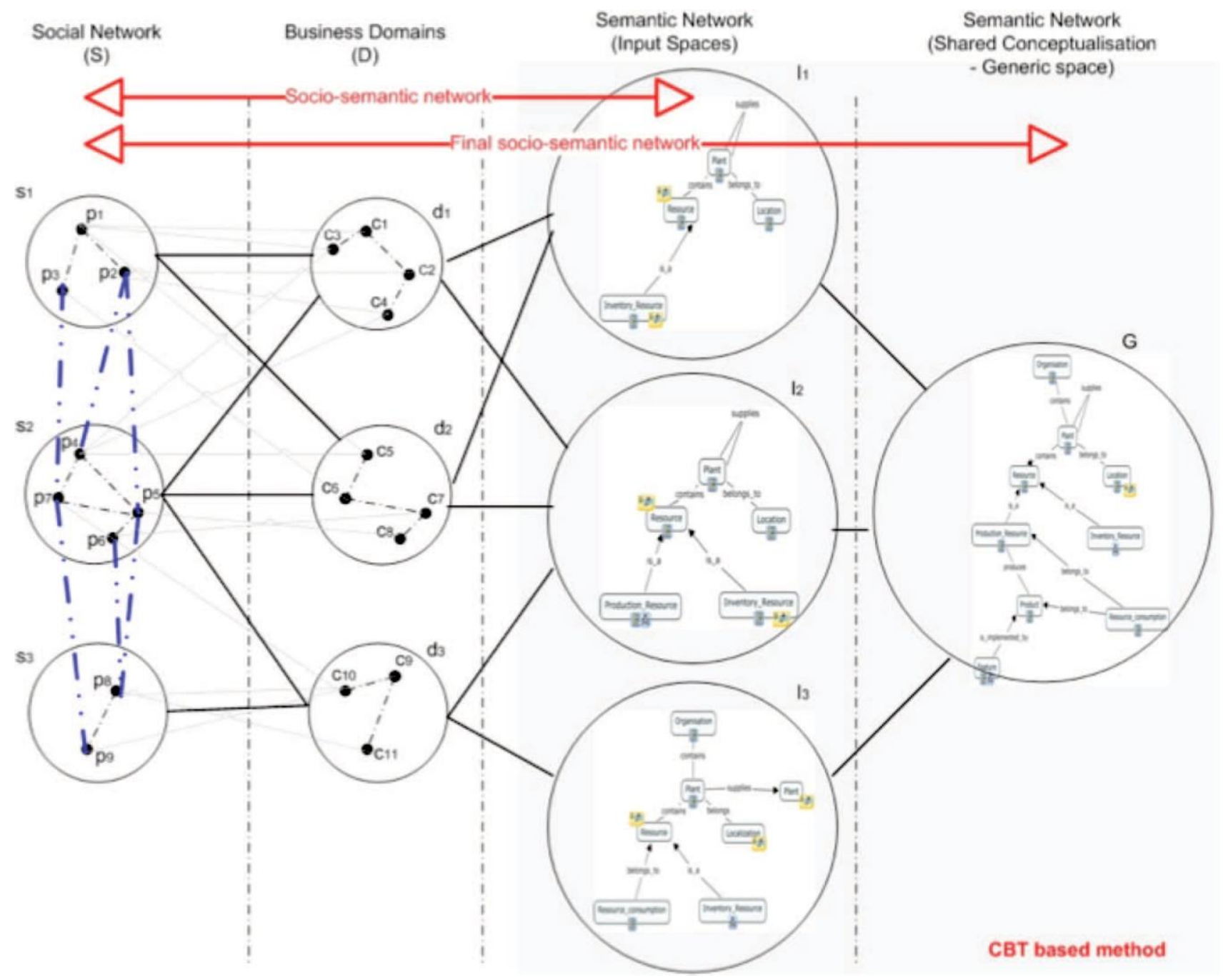

Figure 3. The socio-semantic framework including the CB model.

members (called 'context frame'); (ii) a common ontology with certain goals used in a given time-frame has to be developed and (iii) the common conceptualisation regarding the considered domains, processes and tasks, is the first important collective task that must be undertaken by this team. This approach is only feasible with the support of a tool that facilitates and manages the whole process (see Section 3.3). The proposed method has the following steps (see Figure 4).

Step 0: Based on the definition of the context frame, a subgroup of participants should create a preliminary proposal that will be shared in the generic space and used as the foundations for step 1. This preliminary proposal will be presented using a concept map and it contains the top-level concepts of the domain (this is a starting step and only occurs in the first iteration of the method).
For the following iteration, the shared conceptualisation that is available in the generic space is used as a starting point. The creation of a preliminary proposal is considered the best way to focus a consensus building dialogue (see Susskind et al. 1999).

Step 1: Each organisation has assigned one or more input spaces (only one input space per organisation is considered here for the sake of simplicity) and represents its conceptualisation proposal through the input space. With regard to the definition of 'space', each conceptualisation proposal is a concept map with the information and other knowledge sources (e.g. URLs, documents and other contents) which make the correct understanding of the conceptualisation proposal possible.

Step 2: Using as input the several conceptualisation proposals, the 'counterpart' element is 


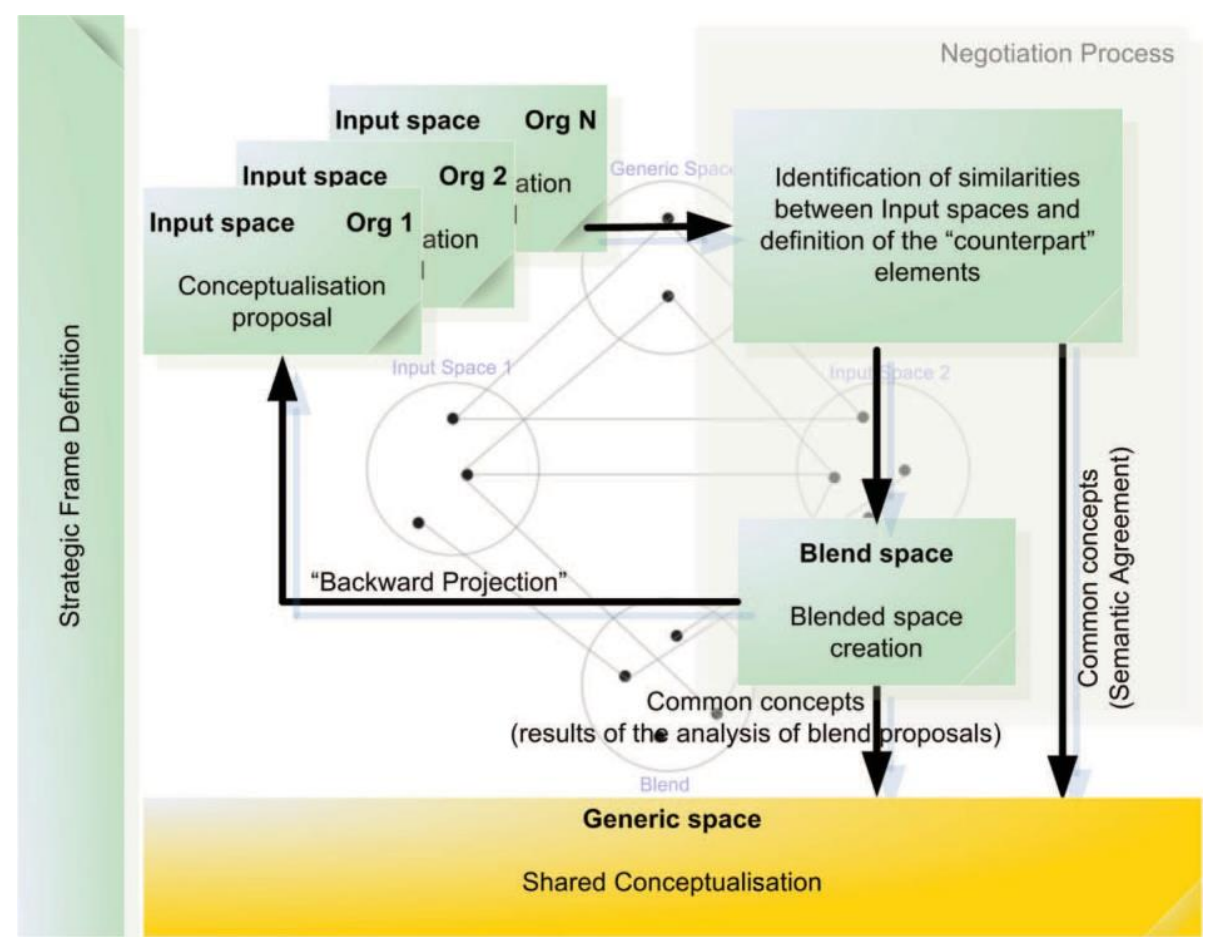

Figure 4. Method to support the collaborative conceptualisation process.

automatically identified using the mapping operation and a proposal for discussion is obtained using the projection operation. This proposal that results from the analysis of the input spaces is presented for discussion in the blend space (the blend space is a 'virtual space', it does not exists directly in the platform, it corresponds to the functions that support negotiation and decisionmaking). The mapping operation returns a set of possible pairs of concepts as a result, this means it analyses the conceptualisation proposals of the various organisations (represented in different input spaces) and identifies a set of mappings between their elements. Based on these mappings between concepts from different input spaces, the projection operation that selects the elements that are projected for the blend space can be performed.

Step 3: The conceptual structure proposed for discussion should be discussed by all members and accepted with minimum negotiation. The parts of the proposal that are accepted by all are 'published' in the shared space (generic space).

Step 4: Based on the input spaces, context frame, generic space and documentation available in the input spaces (called the background information), the blend is 'run' to obtain new conceptualisation proposals (execution of the completion operation). In this step, a new conceptual structure (not presented in the inputs) is proposed. Step 5: New conceptual structures proposed in the blend space are the object of negotiation; the concepts for which consensus exists are represented in ('copied' to) the generic space; situations that justify 'backward projection' are represented in the input spaces and their modifications are analysed (this analysis will be performed by the users, after obtaining a consensus). The emergent structure is then validated (confirm or eliminate new concepts that arise in the blend).

Step 6: If the input spaces are modified, the method should resume at step 2; however, the creation of a new blend space is not necessary.

Step 7: When all participants manifest their agreement with the conceptualisation represented in the generic space, the method instance is concluded.

In summary, at the end of the process, the generic space contains the collective conceptualisation. The blend was used during the negotiation process with the aim of improving, enriching and mainly helping reach a consensus (proposing new concepts, modifying, improving or eliminating concepts). This method may also be used by each organisation to support the creation of its own input space, which can result in the presence of 'multiple blending'. It is important to note 
that in this collaborative process, the validation/ agreement achievement requires each organisation to associate the sources of information which lead to the creation of the input spaces to its input space and justify the proposed content and structure. Whenever an organisation introduces a new concept or relation in their input space, it becomes a target for negotiation. At this moment, the negotiation process is started with the support of the semantic agreement building approach (see Pereira et al. 2009). The network team evaluates the new entrance and if necessary 'runs' steps 2, 3 and 4 to help them make a decision.

\subsubsection{Techniques and tools}

The ColBlend method in its technical implementation encloses two kinds of workflows (core modelling workflows - containing modelling techniques - and supporting workflows). In the supporting workflows, there are the rules that define the procedures needed to enrich the domain modelling process. Information extraction is one of the tasks which were mainly implemented during the creation of blend spaces. However, information extraction could also be used in the other steps in the method presented. Information extraction is automatically extracting relevant terms from a given text corpus, which is usually spread out among the network (documents, web pages, posts, etc.). In order to model a domain of knowledge, it is important to collect a set of domain-relevant terms (term candidates) that constitute the prominent domain-concepts from a linguistic point of view. For this purpose, the TermExtractor tool was used together with the IATE database. The TermExtractor provides a list of relevant terms from an archive of domainrelated documents submitted in any format (doc, pdf, $\mathrm{xml}$, etc.). Afterwards and during the creation of the conceptualisation proposals, IATE was occasionally used to look for the correct term to designate a concept in several different languages. With respect to conceptual representation, concepts maps (supported by CmapTools software) were chosen as the most appropriate technique in accordance with informal domain knowledge representation. The use of concept maps allow inexperienced users who are not prepared to deal with the constraints of formal semantics, to concentrate on the task of constructing a conceptual model. The goal is to enable users to begin building the model informally, without having to commit to a particular knowledge representation and without having to translate their know-how into any particular format. Once the informal knowledge has been built up, its structure may become more obvious. Users could then begin to gradually coerce the conceptual models to conform the formal semantic system. The exploration of CmapTools features leads us to certify some sociotechnical assumptions and specify and/or refine new ones in order to achieve an architecture that is able to support the proposed CBT-based methodology. The CmapTools was mainly used for the creation of the strategic frame and the preliminary proposal and input spaces and the blend and negotiation services were supported by the conceptual modelling environment (ConceptME), which is based on the semantic media wiki (SMW).

The ConceptME is an integrated modelling platform based on two conceptual modules: one supporting the semi-automated construction of the conceptual integration spaces (semBlend) and the other supporting negotiation and consensus reaching (semCons). The ConceptME architecture is defined in three layers (see Figure 5): (1) the content Management layer, where data are located and accessible; (2) the semantic layer, where the data are categorised and organised and (3) the social interaction layer, from where the conceptual models emerge. The social interaction layer

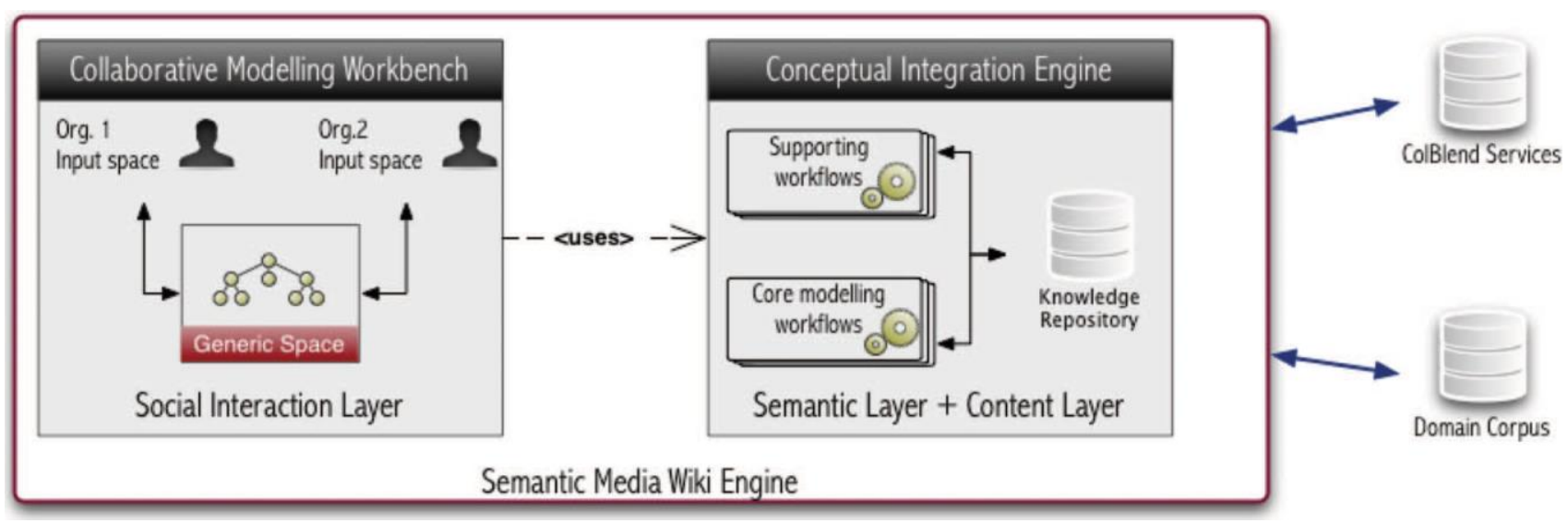

Figure 5. ConceptME platform architecture. 
allow users (all participants in the $\mathrm{CP}$ ) to start the construction of conceptual models by means of domain-specific vocabulary. The semantic level is the core of the conceptual integration engine. At this level, the functionalities that accomplish the operations described by the ColBlend process (core modelling workflow) and the complementary functions which implement supporting tasks (supporting workflows) are implemented. Examples of supporting workflows are: (i) information extraction procedures; (ii) discussion services, promoting the analysis and discussion of the content of the proposals modelled in the social interaction level and (iii) search services, which either check the semantic consistency of the models or inspect the knowledge base and discussion threads for new conceptual evidence. For the core modelling workflow, there is: (i) modelling and ontology management services, using ontology matching or integration algorithms to support the identification of similarities; (ii) import/export services; (iii) graphical conceptual modelling features and (iv) advanced browsing and navigation features. Specific ColBlend functions, which support the workflow procedures were made available as web services. This service-oriented approach makes it possible for further developments in any platform layer without the need to redesign the ColBlend and use other approaches to collaborative conceptualisation construction other than the ColBlend.

The ConceptME currently fully supports the ColBlend process. The use of the SMW engine makes the negotiation of semantic content to reach a consensus easier. By taking advantage of the SMW core features, it was possible to develop mechanisms to discuss, annotate, infer, rate and relate content in an intuitive way. Further services were developed to support integration with other tools (e.g. CmapServer) and standards (e.g. XML and RDF parsing, Web Services, etc.). Moreover, an effort was made to separate content from modelling interfaces and ColBlend core services. The application case described next was accomplished through ConceptME support and CmapTools/CmapServer interaction.

4. Case study: developing an informal ontology in the context of a complex project

\subsection{Project description}

This experiment was conducted within the European project AC/DC 'Automotive Chassis Development for 5-Days Car', in automotive engineering. The vision behind project AC/DC was to provide a vehicle production and supply system that could deliver a customised vehicle within five days, using the experience of leading companies in the European automotive industry. This vision targets not only short order-todelivery lead times and low stocks but also the overall flexibility of the automotive production grid. The approach to achieve this vision is a dynamic supply network system for the automotive supply industry which fully supports the ' $3 \mathrm{Hs}$ ', it is Highly reactive, Highly reliable and Highly flexible. The Customise-toOrder (CtO) principle is the enabler for this new supply network system called 'Dynamic Supply Loops' (DSL). According to the project description, based on CtO principles, the DSL leaves hierarchic production concepts behind, cuts down inventory and ensures $100 \%$ reliability of delivery to the suppliers' customers (Ericsson et al. 2010). A consortium of major European car manufacturers, suppliers and research institutes developed the DSL concept, based on collaborative principles for supply chain management (SCM) which change the conventional automotive terms of delivery to a highly reactive '5-Day-capable' system that radically cuts down inventories in the supply network. This was a large and complex project, as it involved 19 partners from seven countries and nine tasks grouped in three work packages.

In order to develop and disseminate this DSL concept, one of the project work packages aimed to build an ontology that would be used in several project tasks. The general goal of the AC/DC ontology was to facilitate a common and precise understanding of the DSL concepts in the various project activities for all partners. The resulting common vocabulary should match standardised terms in the automotive production domain and the particular concepts (DSL concept) being developed in the project.

\subsection{Before applying the ColBlend method}

The ontology development task initially started without a supporting methodology or even a clear vision of the ontology goals and scope or any debate between the partners. The first obstacle came at this very early stage: where to start and how to start? The first version of the ontology was presented, comprising data models that included concepts, relations, properties and data types. Basically, the first version was based on a software application data model for planning and production scheduling (see Figure 6).

The terms contained in the example above were made available to other members in the form of a 'Prote'ge'Project'. This version came from expert domain partners with no knowledge of the development of ontologies. They were not aware of the real purpose of the "Prote'ge" or its limitations and potential. Therefore, the result did not meet the initial expectations. Moreover, when creating the ontology, the process itself was not very successful, nor was it 


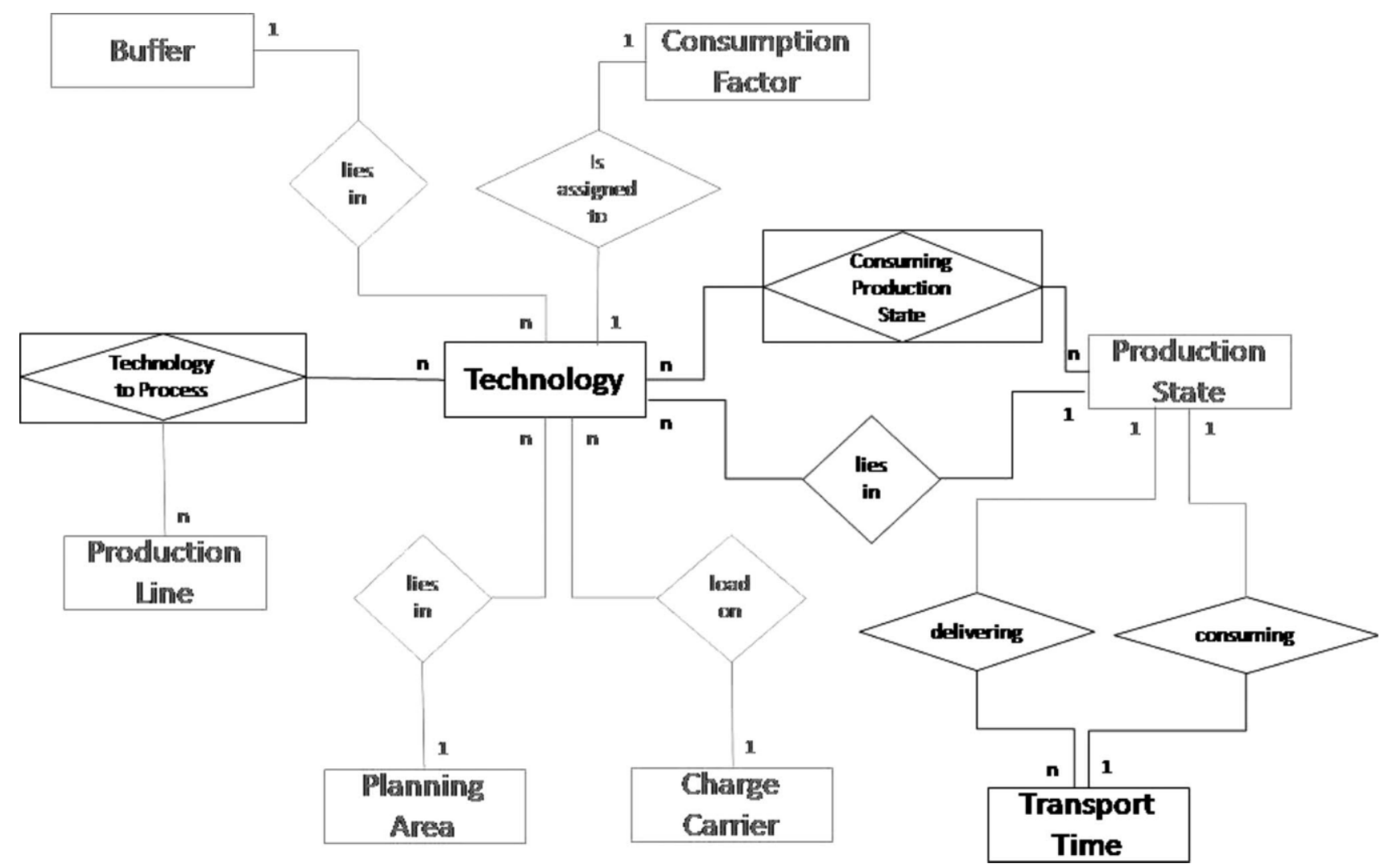

Figure 6. Example of the data model for the production planning process.

truly collaborative or guided by the basic collaborative rules that are essential to support the process. The Prote'ge'files were sent by email to all partners, which, in turn, could reply with comments on the ontology. There was little participation; probably due to the lack of knowledge of the Prote'ge'software and its associated notations, which constituted a barrier, making the process move forward without contributions from all those involved.

\subsection{Application of the ColBlend method}

Within this context, an action-research project was set up, aiming to help the project develop its ontology and also, create knowledge on the collaborative construction of ontologies by designing and undertaking a set of experiments using the ColBlend method and the ConceptME platform.

\subsubsection{Situation analysis and experiment design}

It was important to see whether it would be possible to reuse existing ontologies in SCM domains. In the ontologies studied, Ureten and Ilter (2006) and Fayez et al. (2005) were based on the SCOR model; others such as Maier et al. (2003) focused on inside organisations but not among them and 'The United Nations Standard Products and Services Code' which provides an open, global multi-sector standard for the efficient and accurate classification of products and services. These ontologies cover very specific subdomains, making them difficult to reuse. There are also several upper-level ontologies that are too abstract to be applied in specific situations. Since the collaboration concepts behind supply chains and their requirements could be fundamentally different, there is no standard ontology which would be detailed enough to be applicable in every practical case.

\subsubsection{The context of the experiment}

The application case scenario was identified and some preliminary studies were conducted, leading to the following experiment: Four teams from four different organisations (geographically dispersed) participated in the domain conceptualisation. Two of these teams (Team1 and Team2) from two different organisations (Org1 and Org2) were domain experts from academic and professional areas, respectively; another team (Team3) from another organisation (Org3) was composed of experts in information and knowledge management and in collaborative networks; the 
fourth team (Team4) from another organisation (Org4) in addition to their academic expertise in the specific domain, had a reasonable understanding of ontologies.

The roles of each actor were (i) the Contributor: all team members should play this role, contributing to the improvement and enlargement of the current version of the AC/DC conceptualisation. The responsibilities of the contributor are to offer input on the shared conceptualisation by proposing and discussing concepts and relationships and (ii) the Facilitator: this person is responsible for facilitating the discussion/ negotiation of the conceptualisation.

All teams were assigned the role of contributor and one team member was selected to assume the role of facilitator. This was chosen by all participants in the process in accordance with their expertise in the management of CmapTools and ConceptME.

\subsubsection{ColBlend method implementation: Context frame}

It was decided that initially the ontology would contribute to a common understanding of the concepts used and needed by all partners in the various project activities, their goals may be expanded in the next iterations of the process. Therefore, considering the original purpose, it was agreed that the first instance of the ontology should reorganise itself around the main concept (DSL concept), showing the organisational levels and the implied top-level processes and activities. In addition to domain structuring through capturing, organising and justifying the terms from the DSL root concept, it was necessary to identify the currently well- known concepts in SCM, which when combined with the not so common DSL concepts, will help understand and define this new approach.

\subsubsection{ColBlend method implementation: initial shared conceptualisation}

With the main focus on the dynamic supply chain collaboration concept, more precisely the DSL concepts, a subgroup of team members created an initial shared conceptualisation, which included the high-level concepts associated to 'DSL', guided by the following focus question: 'what processes, activities and information are involved in the DSL network planning model, making collaboration possible in the entire supply chain in feedback loops?' The result was presented in a concept map and made available to the entire team to serve as a starting point for the creation of their conceptualisation proposals. Given the large number of processes and activities associated with the concept, 'DSL' (see Figure 7), the CP was divided into several iterations where the different processes would be addressed. This organisation followed the way in which the DSL concept was decomposed and internally developed in the project. The DSL operates on two levels: on the network level and the manufacturing plant level. When talking about the DSL, this implicitly means planning, which means that the collaborative planning methods occur in $3 \mathbf{k} 1$ levels, three typical levels of management (operational, tactical and strategic) and one more, the transversal level, which is associated with event management in the supply chain. Each loop comprises a set of processes with specific activities.

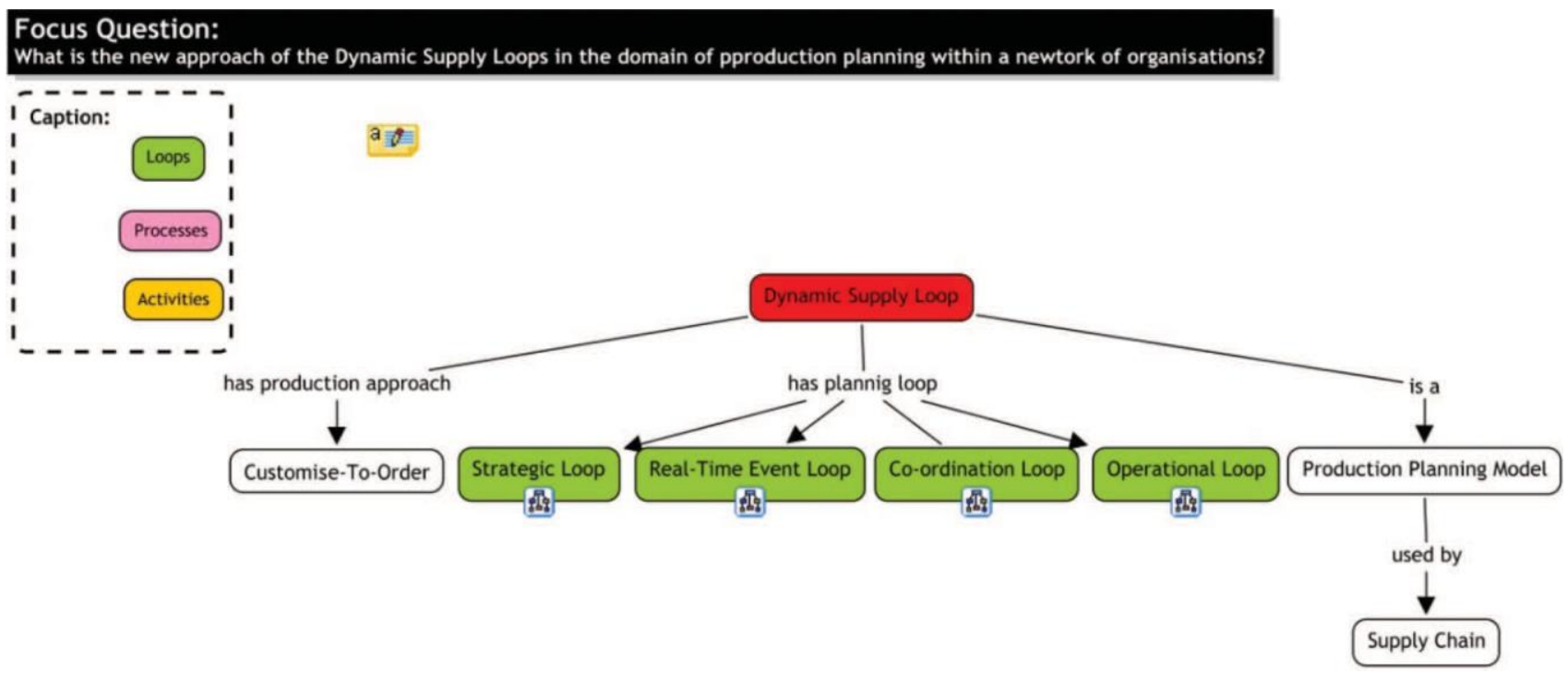

Figure 7. High-level map for the 'Dynamic Supply Loops' concept. 
The first process addressed was the 'Production Planning Process', which is part of the 'strategic loop' and 'operational loop', shown in Figure 7. A concept map was also built for this process as a preliminary proposal (see Figure 8). For reasons of readability and confidentiality, only excerpts of the resulting maps are shown.

\subsubsection{ColBlend method implementation: conceptualisation proposals}

After the construction of the first concept map (the preliminary proposal, see Figure 8), an object was created in ConceptME in order to aggregate ideas and propositions for the Production Planning Process. Consequently, all of the teams in the project were able to extend the first shared domain conceptualisation, contributing with their own inputs (changes that were made in the input spaces in CmapTools or ConceptME) which were published. Figure 9 portrays the conceptualisation proposals created by two of the organisations involved. Each member could publish its proposal in the shared space and this was viewed by all members.

Using each team's domain expertise, new inputs were created from the results from; the TermExtractor tool document analysis, other documents produced by the various project work packages, the logistics section of the SAP dictionary, the terms and glossaries on SCM proposed by Vitasek (2008) and most importantly, the identification of the conceptual structures in the minds of the experts when they interact with the other member's proposals.

\subsubsection{ColBlend method implementation: conceptualisation proposal analysis (input spaces) and creation of a proposal for negotiation}

It had already been decided during the negotiation process that a proposal would be created for negotiation based on the two input spaces that were considered most complete (step 2 of the ColBlend method, mapping operation and the selective projection operation). An excerpt of the proposal obtained for negotiation is shown in Figure 10. The discussion of this new proposal was initiated using the following features of the ConceptME: semantic annotation; rating and voting mechanisms; suggestion mechanisms and inferencing, outlined in Section 3.3. At the end of the discussion, the approved (consensual) concepts are published in the shared space, where they can be consulted by all members.

\subsubsection{ColBlend method implementation: the generation of new elements for negotiation}

In this experiment, steps 4 and 5 of the method ('execute' the blend and analyse their proposals) were simulated as there is no support yet in ConceptME for these steps. Based on the measures 'term weight' and 'semantic similarity' and using the TermExtractor software, new elements were proposed for inclusion

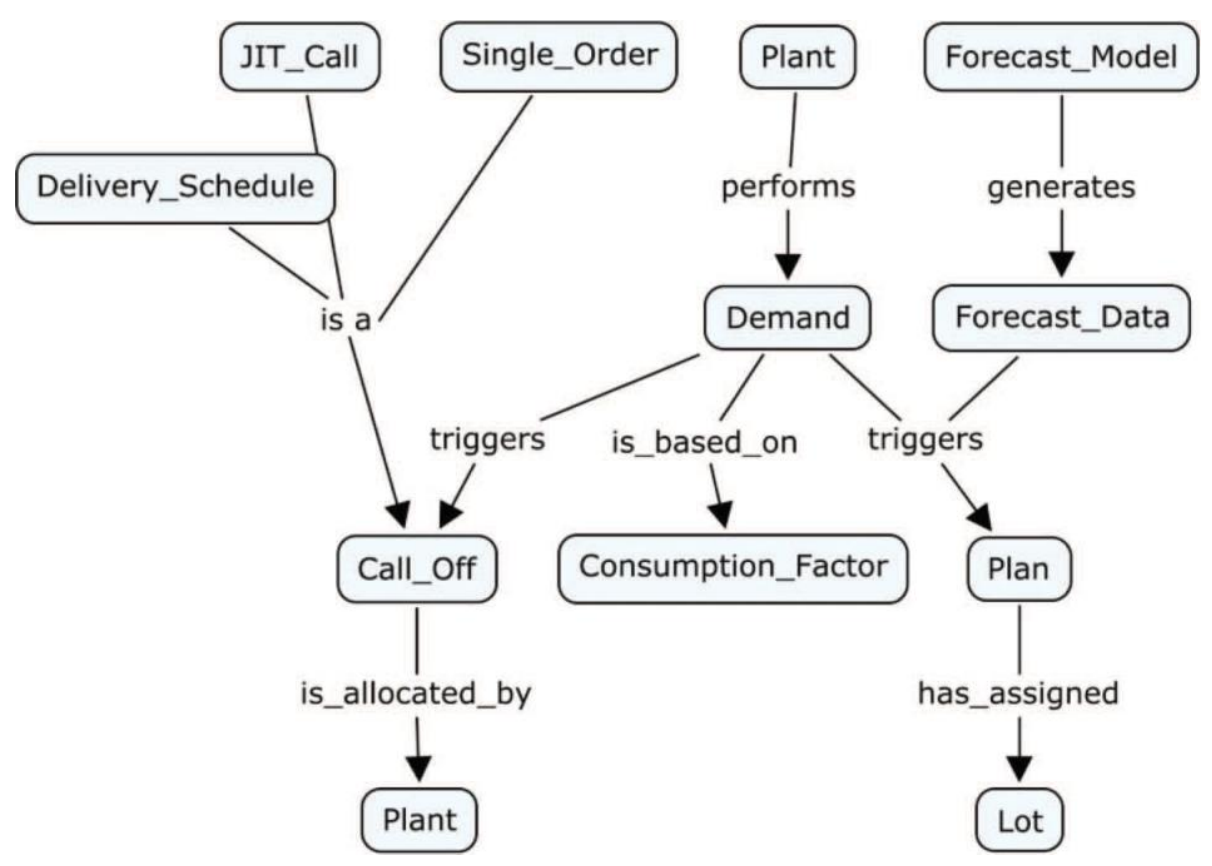

Figure 8. Preliminary proposal for the 'Production Planning Process'. 


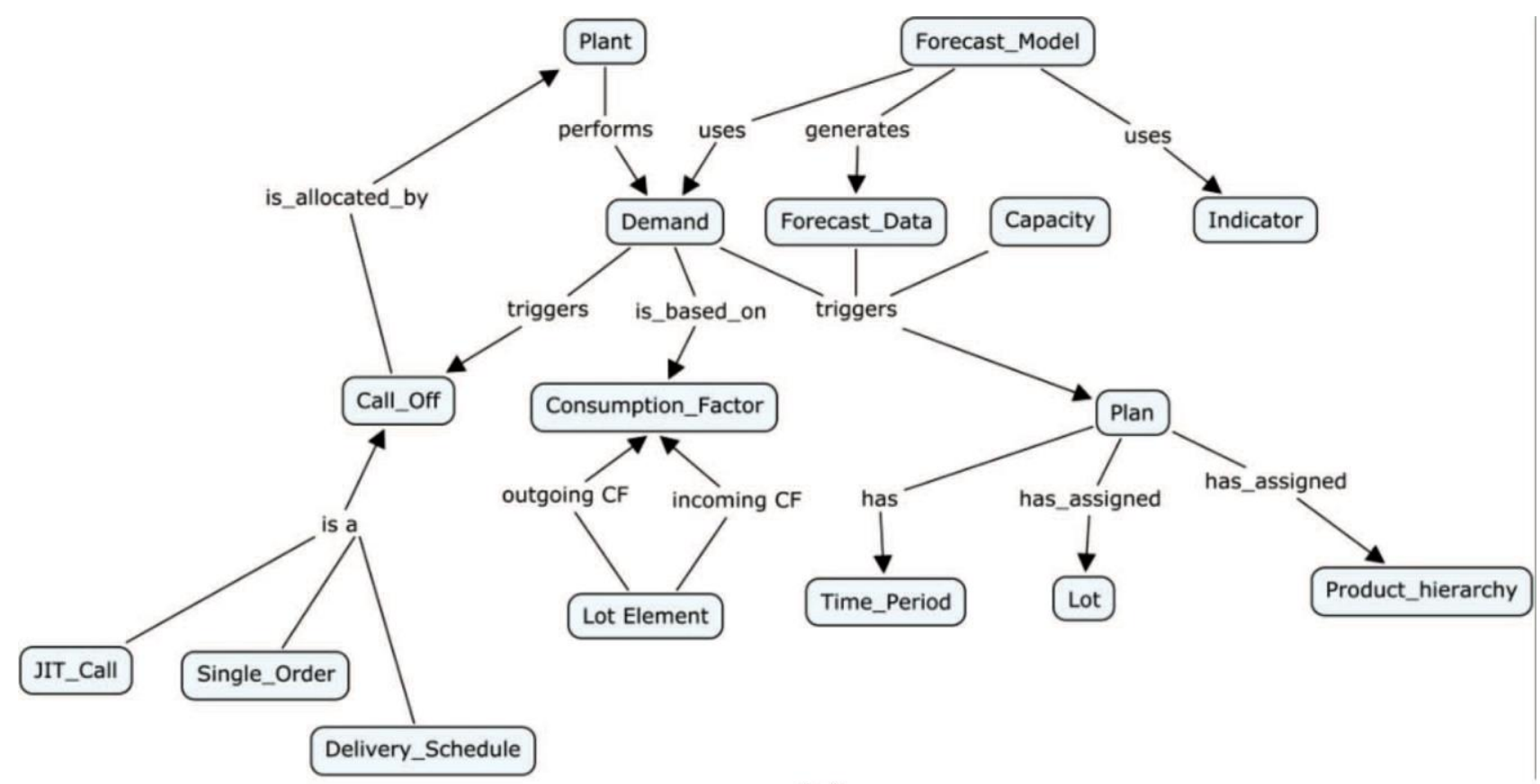

(a)

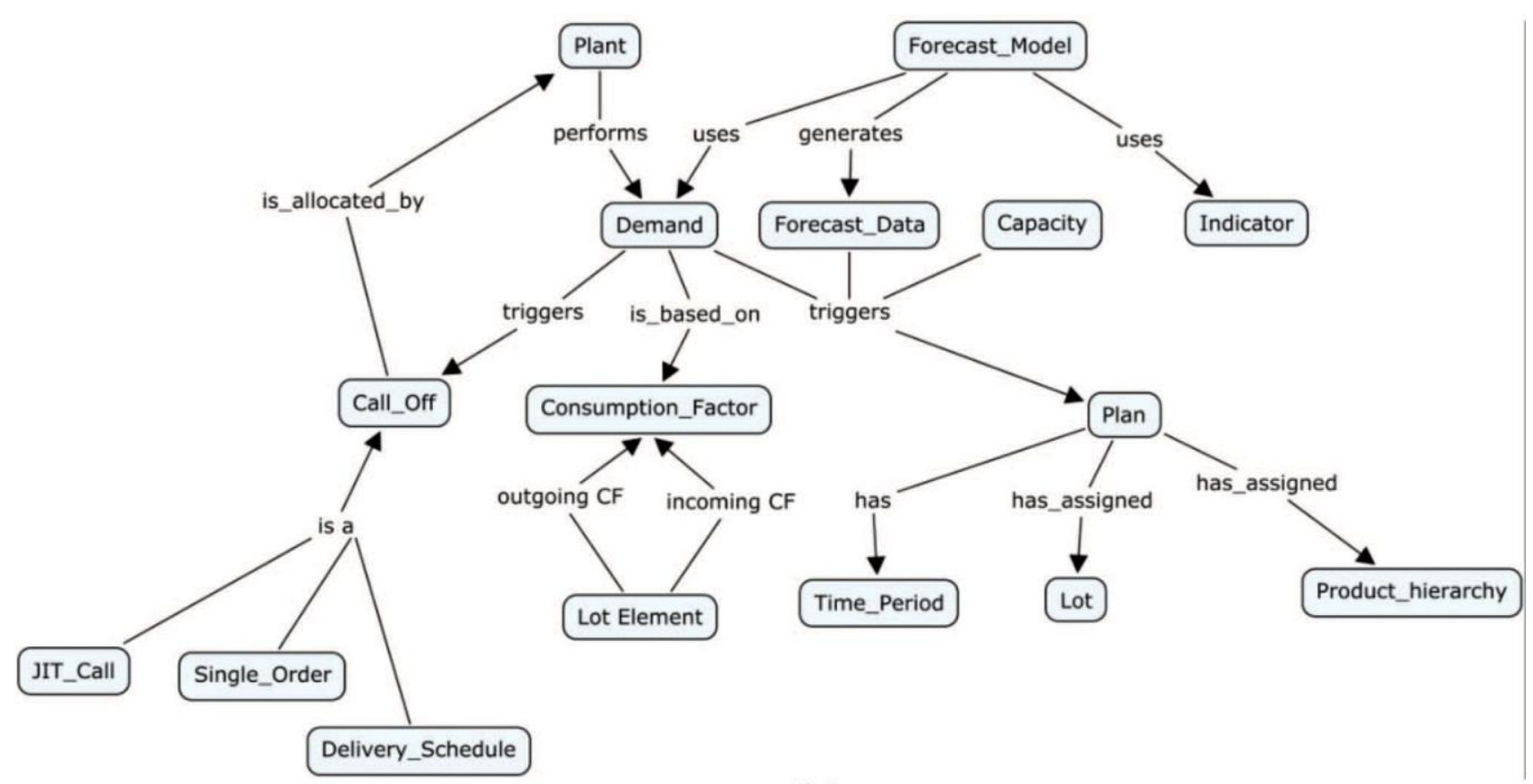

(b)

Figure 9. (a) Partner conceptualisation proposals (examples of input space - 1). (b) Partner conceptualisation proposals (examples of input space - 2).

in the shared conceptualisation. The execution of the completion operation was simulated, this corresponded to the presentation of new conceptual structures that were not present in any of the input spaces. Some of the terms proposed by the
TermExtractor, with 'weight' that is equal to or greater than 0.5 were: product hierarchy, BOM, plan, frame plan, sequence plan, strategic loop, operational loop, cost estimates, forecasting method, supply network, tactical loop, demand prediction, plant level, planning 


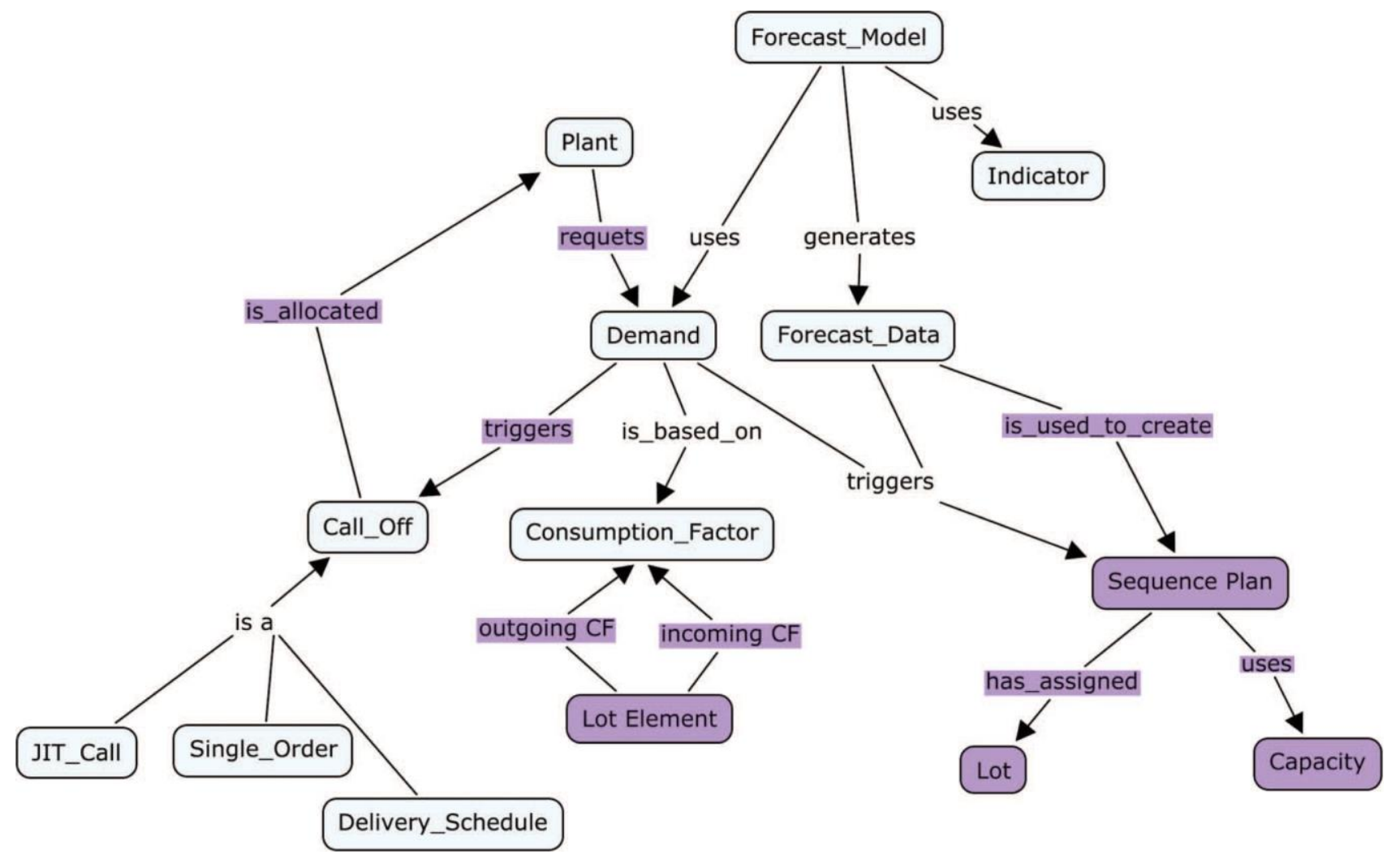

Figure 10. Excerpt of the proposal presented for negotiation.

horizon, product family and collaborative demand prediction. From this list of terms, those that were already in the shared proposal were ignored and all terms that were not in the proposal and had 'weight' that was equal to or greater than 0.5 were considered candidates. Of the candidates, the terms with a semantic similarity value that was equal to or greater than 0.5 were proposed for discussion.

\subsubsection{ColBlend method implementation: shared conceptualisation}

After two iterations of the method, which included the presentation by participants of new elements and the generation of new conceptual structures based on term extraction and features from the negotiation process (conducted at SMW), the final result for the 'production planning process' is presented in Figure 11 for the operational and strategic levels.

\subsubsection{ColBlend method implementation: technical support}

Technically, all of the process steps are supported by the CmapTools and ConceptME platforms. The CmapTools aggregates all of the input spaces on the server; therefore, users are able to publish them in
ConceptME. With TermExtractor, the enrichment of the current conceptualisation was achieved through the discovery of new terms and the supported validation of existing ones. All of the input spaces have the same conceptual structure and the person in charge of coordinating the process uploads the final (consensual) map onto the server. The concept maps presented on the CmapServer comprise the generic space.

\subsection{Main observations and results \\ 4.4.1. Benefits in the automotive industry of the ColBlend method (conceptual models and ontology development)}

The DSL specifies a coordinated process for planning and controlling supply networks while minimising planning effort and communication overheads. The DSL method encompasses five basic principles that incorporate faster and more flexible network planning and operation (Ericsson et al. 2010): (1) descentralised planning; (2) tier-to-tier communication only; (3) collaboration principles; (4) CtO using customerneutral parts and (5) demand-oriented planning. By using the DSL, the traditional hierarchical automotive supply chain planning concept will be modified to establish a one-step feedback planning loop between 

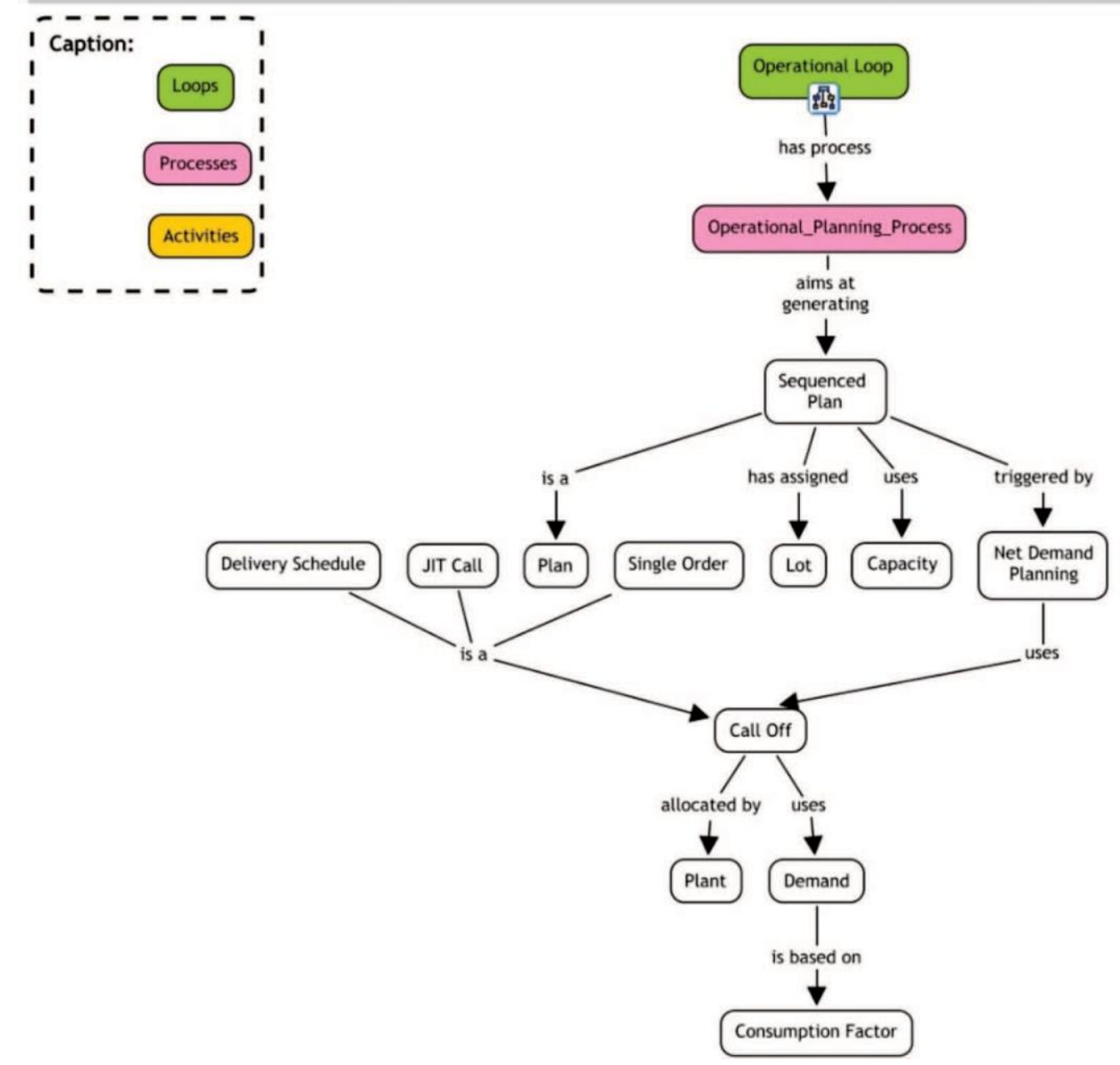

Figure 11. Excerpt of the concept maps created during the conceptualisation process.

tier $n$ and tier $\boldsymbol{p l} 1$, between any OEM and/or supplier. Following these leads, the AC/DC ontology was developed to ensure interoperability between enterprises on three levels: (i) the infrastructure; (ii) the protocol which determines what and when to communicate and (iii) the conceptual level which defines a common dictionary.

Accordingly, a set of necessary messages was identified and typified referring to framework plans, supply and demand information on long-, medium- and short-term horizons, call-offs, cost-estimations, inventory reports and shipping notifications, for example, and they were implemented in the AC/DC messaging service. As depicted below (see Figure 12), the AC/DC ontology was an important means for identifying the messages needed to support this new collaborative approach of planning and controlling supply chain networks. The example shows three fundamental messages that implement the frame planning loop, namely: getCostEstimates, frame plan scenario (FPS) cost estimates and inform FP. The getCostEstimates messages (for example) aims to query the suppliers to provide cost estimates for parts and logistics according to a certain delivery rhythm. The FPS cost estimates is a return message from thesupplier to thecustomer. It determines the costs for the parts and logistics in the suggested delivery rhythm and min-/max-demands per quarter.

Figure 13 shows a more dynamic view of the message flow. Moreover, an example of the main data for the getCostEstimates/FPS cost estimates (request/ response) messages is shown. This kind of specific information was identified afterwards; nevertheless, the ontology was the main agent that triggered messages and content identification.

Afterwards, a flexible and alternative messaging service was provided where all of the identified 


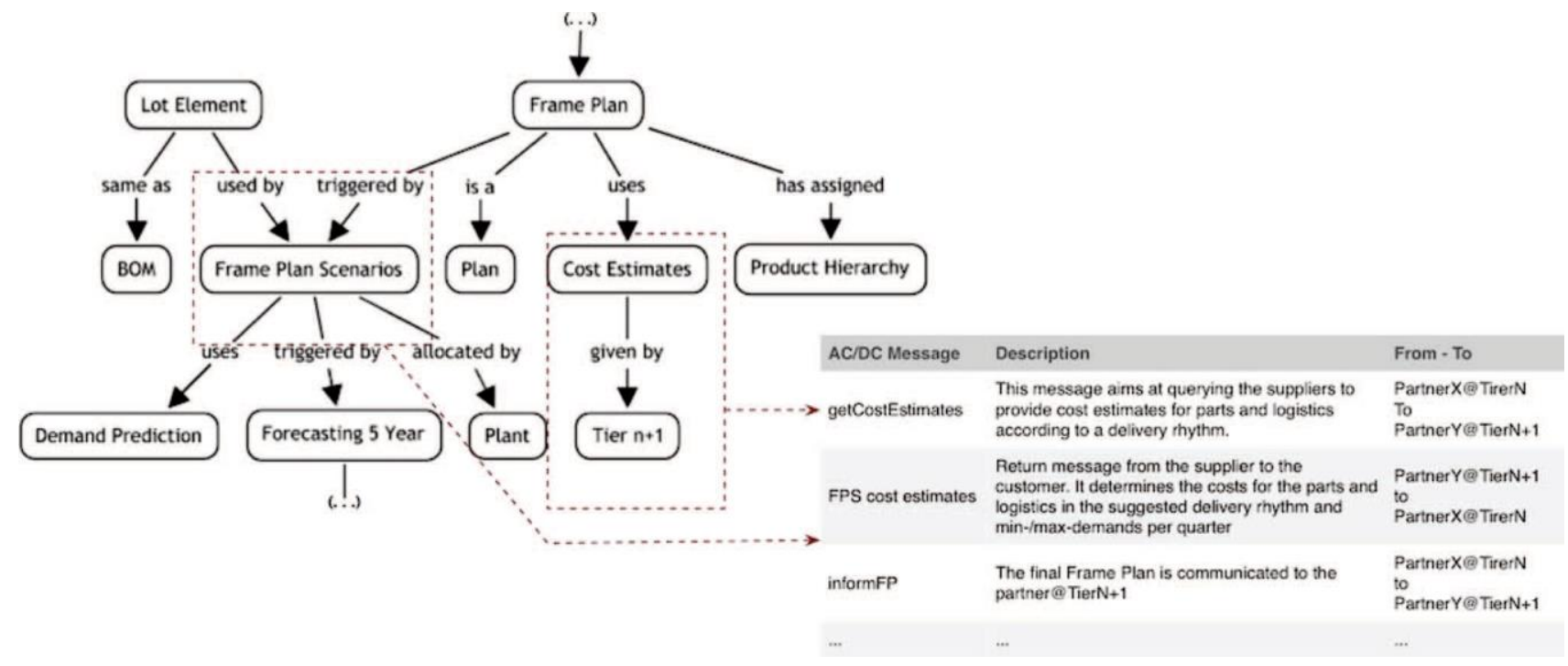

Figure 12. From AC/DC ontology to Message Specification.

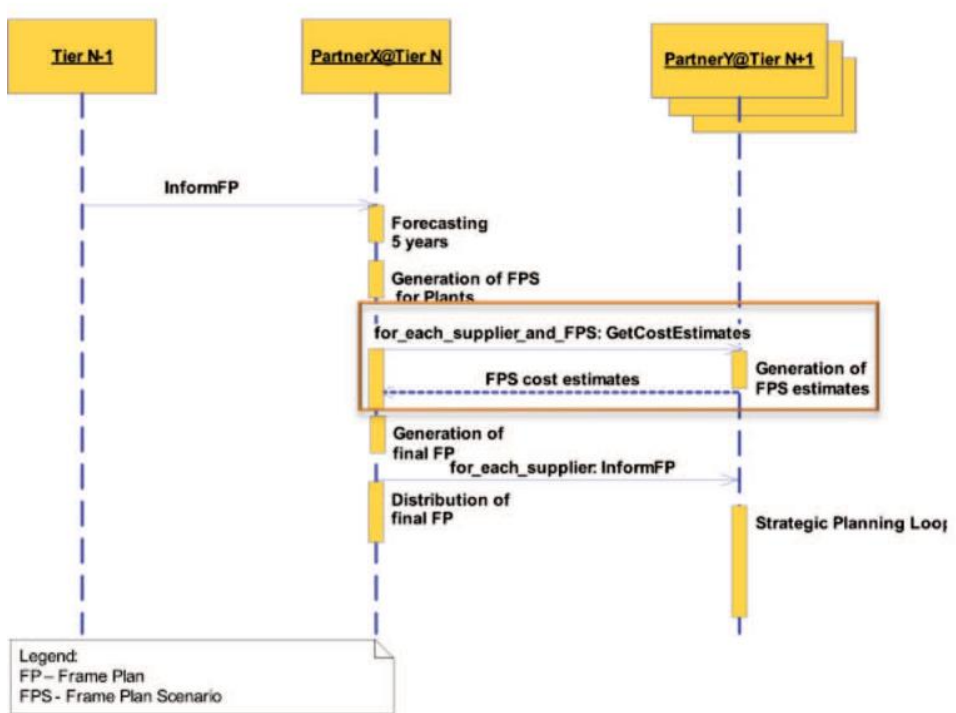

\begin{tabular}{|c|c|c|c|c|}
\hline & \multicolumn{4}{|c|}{2009} \\
\hline & Q1 & Q2 & Q3 & Q4 \\
\hline Del. Rhythm & weekly & weekly & weekly & weekly \\
\hline Min demand & & & & \\
\hline Max demand & & & & \\
\hline Min Capacity & & & & \\
\hline Max Capacity & & & & \\
\hline Locations & & & & \\
\hline & \multicolumn{4}{|c|}{2009} \\
\hline & Q1 & Q2 & Q3 & Q4 \\
\hline Del. Rhythm & weekly & weekly & weekly & weekly \\
\hline Min demand & & & & \\
\hline Max demand & & & & \\
\hline Min Capacity & & & & \\
\hline Max Capacity & & & & \\
\hline Locations & & & & \\
\hline Productioncost & & & & \\
\hline LogisticalCost & & & & \\
\hline
\end{tabular}

Figure 13. getCostEstimates (request/response) example.

messages were implemented. In this context, an application programming interface (API) is coupled with the company's enterprise resource planning (ERP) system as an automatic communication system. This API can transmit and receive messages. Yet, based on conceptual models created using ColBlend, a common dictionary was defined. This dictionary makes software systems that are at different locations compatible since it contains a set of typified messages (mutually agreed) and the subsequent flow of activities and tasks are commonly understood by each party.

In addition to providing a common platform for communication despite different in-house terms and vocabularies, the ontology is of great assistance when it comes to company mergers, which occurred during the project, to reach a common understanding and 
achieve expected synergies more efficiently. It also (Ericsson et al. 2010) allows future users of the AC/DC results to comprehend the knowledge behind the DSL processes and extend it with formal inference methods, if necessary. As a consequence, through the use of these new mechanisms by all partners in the network (collaboration), the overall process of adoption is improved (speed), it is more reliable and flexible (flexibility) and it works with reduced cost for all participants in the supply chain.

The overall balance of the benefits resulting from the definition and operationalisation of the DSL concept were (Ericsson et al. 2010): (1) It ensured better collaboration between the partners in the supply network and enhanced speed and flexibility as well as process reliability over the whole supply grid; (2) It provided great flexibility in production systems; (3) The production system was capable of managing $+25 \%$ capacity fluctuation per day, without increasing costs; (4) The strategic objectives achieved using AC/DC principles in the supply network: lead time reduction of up to $85 \%$; inventory reduction of up to $50 \%$; operator reduction of up to $8 \%$; floor space reduction of up to $6 \%$; defect reduction of up to $50 \%$ and flexibility per day $+25 \%$. Although the ColBlend approach was not the main agent of this success, it can be reliably concluded that the contribution provided for the clarification and dissemination of the fundamental concept became a key factor in the overall results.

Another interesting fact was that the work performed under project AC/DC, using the ColBlend method and associated tools, caught the attention of the remaining project partners, especially the project leader company, for the process and its benefits in creating semantic artefacts. This partner is a growing organisation and recently acquired other companies. One of the biggest challenges that it has faced has been the implementation of their processes and knowledge sharing procedures associated with the operationalisation of them. The company developed this process using concept maps as a simple way of providing information about their processes and sharing how they are implemented. The technological framework (described in Section 3.3) inherent to the experiment conducted under this project also served as an inspiration for the company to start its new approach to knowledge sharing. This reveals the satisfaction of the project leader with the procedures used during the construction of the informal ontology.

\subsubsection{Evaluation of the practices associated with the} collaborative conceptualisation process

One of the foundations of the CCP is to let users express their knowledge on an environment without constraints. This is achieved, providing users with the appropriate methods and tools to perform their modelling tasks. Nevertheless, the unawareness of these methods and tools could lead to an inadequate choice. For example, the type of players and roles in the process, knowledge areas, technologies and techniques proposed are key factors that may be decisive for the success of the overall process. Concept maps, for instance, was the graphical tool used to organise and represent domain knowledge. The Concept maps appeared to be suitable within a CCP, as they revealed the great acceptance by the users and because in this phase 'completeness is more important than neatness and rigor' (Eskridge et al. 2006). From our observation and from the interviews, it can be concluded that discussing the problem using a domain-specific vocabulary supported by a visually oriented, easy to use, informal tool, provided effective results in a relatively short period of time. Our goal was to allow users to informally start the construction of a 'knowledge model' without having to commit to a particular knowledge representation and without having to translate their know-how into any particular knowledge representation format. After the conceptual model has been constructed, its structure may become more clear and useful. Therefore, users could then begin to gradually coerce the concept maps to conform to the formal semantic system. The following paragraphs share some lessons that were learnt with this experiment.

(1) Initial social and cultural analysis: The social and cultural analysis of the various partners, although brief, is necessary to design the process. For example, the decision to follow a process with rules and procedures that are more or less rigid may depend on the type and number of players in the process. In the $\mathrm{AC} /$ DC project, due its size and heterogeneity (19 partners, from seven countries), some formal rules and procedures had to be defined to ensure coordination during the conceptualisation task: an initial formal meeting was conducted to prepare the process (select a mediator; identify relevant participants; define work rules, define an agenda, define roles for each participant), to define the goal, scope and boundaries of the conceptualisation task and the tools that would be used.

(2) Appropriate definition of the context frame and road map: The starting point for this case study clearly shows the importance of these tasks. After the initial definition of the context frame and road map, it is equally important that in the previously specified time periods, go 
backwards and review the following questions: 'What do we have? What do we want? and How do we get there?'. These questions allow the team to evaluate the strengths and weaknesses of the current situation. The definition of the context frame and road map was executed in the first formal meeting and were adjusted as the process moved forward. This was a fundamental task to define the boundaries of the conceptualisation (what is inside and what belongs to your environment).

(3) Rules to organise the process and motivate the participation: The evaluation showed that the majority of users were passive in their participation. Automatic notifications of all teams whenever changes exist, version control and definition of a time frame in which the proposals can be discussed are fundamental to organise the process better and motivate the participants more. Therefore, if no-one presents suggestions during the time period defined, it means that an agreement exists. Every time there is a change in any discussion item within the process, users should be informed and invited to comment on the new proposals. All of these procedures must be agreed initially. Also in an early stage, a strategy that will lead the negotiation process should be defined (a combination of working with a single-document text and taking a vision approach was used, for details see Pereira 2010). The use of workflow mechanisms makes it possible to manage the process and maintain control of the various conceptualisation proposals that are created.

(4) Always try to reduce the complexity of the process: The collaborative process of conceptualisation is really complex because of the high number of areas, processes and activities, for example. One way to deal with this, regarding the visual knowledge representation is to follow rules, such as Kremer (1994) or GómezGauchía et al. (2004b): create small concept maps with a few concepts and relations, organise them in an orthogonal way with horizontal and vertical levels of abstraction, organise them by subarea of ontology and use a colour-based code.

(5) Project generated documentation as an enabler: The continuous production of project documentation is a way to validate and improve the conceptualisation. On the other hand, the consensual conceptual structure agreed so far, should be used in the production of new deliverables in order to standardise the contents of each deliverable. Therefore, it is easy to share and understand the meaning of the concepts in the domain (Soares et al. 2000). For example, to create the first version of the conceptualisation, the documentation for the AC/DC proposal was used. During the edition/ creation of the deliverables, an attempt made to use and validate the contents according to the concepts defined in the conceptual models created.

(6) The first version of the conceptualisation: The necessity of the preliminary proposal was identified in the first experiment. Even with only the high-level concepts, this first version proved to be a good way to focus the discussion and guide the process. This preliminary proposal was developed by a subgroup of participants based on the documentation associated with the AC/DC project proposal. The preliminary proposal should focus on the points defined in the agenda and provide the widest range of ideas and options. The proposal was used to focus the CP.

(7) The existence of a negotiation space (blend space): The existence of a blend space provided more reliability, collaboration and agility to the $\mathrm{CP}$. This was due to the fact that the inputs for the blend were based on documentation produced in the project, as well as other important resources selected by the domain experts. The proposals that were created in an automated way, using the mapping, projection and completion operations showed a high level of acceptance.

(8) Carefully selecting the information resources used as inputs in the blend space: The results obtained in the blend directly depend on the information sources used. The blend results can be accepted with more or less support, according to the inputs provided during its creation.

\section{Conclusions and future work}

The approach advocated in this article proposes a shift in the process of creating semantic artefacts from an 'artefact engineering' perspective to an 'actorartefact co-evolution' perspective. The colla- borative construction of shared conceptualisations is the great challenge, particularly in the context of networks of organisations (including manufacturing networks), where this problem had not been ad- dressed. There are no methods to support general conceptual negotiation processes besides the concep- tual blending method applied in the case described in this article. 
The theoretical and conceptual framework described here (the formal part is described in a forthcoming paper) can be useful in the analysis of collaborative networks in manufacturing (for instance, supply chains, complex project partnerships). Furthermore, the authors believe that the added value of this article is in the detailed description of the case study with regard to the development of a new set of interrelated complex concepts derived from the SCM domain (DSL). The action-research project that was established by our research team aimed to solve the problem of managing the development of a new set of concepts by an heterogeneous set of actors and simultaneously improving the results of our research in this field. It allowed us to: (i) obtain more knowledge on the collaborative $\mathrm{CP}$ in real application scenarios; (ii) to evaluate and improve the ColBlend method and (iii) to improve the design of the ConceptME platform that supports all of the steps in the ColBlend method. Although the ColBlend method and associated tools can be applied to collaborative networks in general, the main areas in manufacturing where this approach has great potential are: (i) collaboration and communication in SCM, for example, to develop a common metadata structure to annotate (tag) the messages exchanged in the chain; (ii) information organisation in a supply network, for example, by using the method to develop a common content and document organisation structure to share between the client and their suppliers and (iii) knowledge management and coordination of activities in an innovation network, for example, by helping to conceptualise new ideas, creating new concepts and conceptual structures.

After these experiments had been conducted in the case study and seeking the continuous improvement of ColBlend and ConceptME, our perspectives for future work include: (i) developing a formal framework including multi-modal corpora in a socio-semantic network model (the current model can only include concept maps), using knowledge of terminology and computational linguistic areas; (ii) solving the problem associated with the interpretation of the conceptual models created, since they may be ambiguous. One possible way of ensuring a common interpretation of the conceptual models created will include sharing a set of basic top-level conceptual structures and the meaning of their concepts and relations. The (re)use of these pre-defined conceptual structures could, on the one hand, 'unlock' the initiation of the CP and, on the other hand, reduce the ambiguity of the knowledge structures; (iii) applying social network analysis to study the influence of social relationships in the meaning negotiation process and (iv) extending the
ColBlend method to support the development of multilingual conceptualisations.

\section{Acknowledgements}

This work is funded by the ERDF through the Programme COMPETE and by the Portuguese Government through FCT - Foundation for Science and Technology, project PTDC/EIA-EIA/103779/2008 'CogniNET'.

\section{References}

Aschoff, F., 2004. Knowledge mediation: a procedure for the cooperative construction of domain ontologies. Master's thesis, University of Heidelberg.

Breslin, J.G., et al., 2010. Semantic web computing in industry. Computers in Industry, 61 (8), 729-741. doi:10.1016/j.compind.2010.05.002.

Camarinha-Matos, L.M. and Afsarmanesh, H., 2005. Collaborative networks: a new scientific discipline. Journal of Intelligent Manufacturing, 16, 439-452.

Camarinha-Matos, L.M. and Afsarmanesh, H., 2007. A framework for virtual organization creation in a breeding environment. Annual Reviews in Control, 31, 119-135.

Chungoora, N., Canciglieri, O., and Young, R., 2010. Towards expressive ontology-based approaches to manufacturing knowledge representation and sharing. International Journal of Computer Integrated Manufacturing, 23 (12), 1059-1070. doi:10.1080/0951192X.2010.518976.

Corcho, O., Ferna'ndez-Lo'pez, M., and Gómez-Pe'rez, A., 2003. Methodologies, tools and languages for building ontologies. Where is their meeting point? Data \& Knowledge Engineering, 46 (1), 41-64.

de Moor, A., Leenheer, P.D., and Meersman, R., 2006. Dogma-mess: a meaning evolution support system for interorganizational ontology engineering. In: Proceedings of the 14th international conference on conceptual structures (ICCS 2006), 17-21 July, Aalborg, Denmark, LNAI 4068. Berlin: Springer Verlag, 189-202.

Ericsson, R., et al., 2010. From build-to-order to customizeto-order: advancing the automotive industry by collaboration. In: R. Ericsson, et al., eds. Code of practice findings of the EU-FP6-Project AC/DC - automotive chassis development for 5-days cars. Go"teborg: Consortium of the AC/DC Project and modularity.

Eskridge, T., et al., 2006. Formalizing the informal: a confluence of concept mapping and the semantic web. In: A.J. Can ${ }^{\sim}$ as and J.D. Novak, eds. Concept nos theory, methodology, technology. Proceedings of the second international conference on concept mapping (Vol. 1). San Jose, Costa Rica: Universidad de Costa Rica.

Evans, V. and Green, M., 2006. Cognitive linguistics: an introduction. Edinburgh: Edinburgh University Press.

Fauconnier, G. and Turner, M., 1998. Conceptual integration networks. Cognitive Science, 22 (2), 133-187.

Fauconnier, G. and Turner, M., 2002. The way we think, conceptual blending and mind's hidden complexities. New York: Basic Books.

Fayez, M., Rabelo, L., and Mollaghasemi, M., 2005. Ontologies for supply chain simulation modeling. In: Proceedings of the 37th winter simulation conference, 4-7 December, Orlando, FL, USA. New York: ACM, 23642370. 
Ferna'ndez-Lo'pez, M. and Go'mez-Pe'rez, A., 2002. Overview and analysis of methodologies for building ontologies. The Knowledge Engineering Review, 12 (2), 129-156.

Go'mez-Gauchı'a, H., Di'az-Agudo, B., and Gonzalez Calero, P.A., 2004a. Towards a pragmatic methodology to build lightweight ontologies: a case study. In: Proceedings of the IADIS international conference, Applied Computing 2004, Lisboa, Portugal. ISBN: 972-98947-3-6.

Go'mez-Gauchı́a, H., Dı́az-Agudo, B., and Gonza'lez-Calero, P., 2004b. Two-layered approach to knowledge representation using conceptual maps and description logics. Concept maps: theory, methodology, technology. In: A.J. Cañ as, J.D. Novak and F. M. Gonza'lez, eds. Proceedings of the first international conference on concept mapping, Vol.1. Pamplona, Spain: Universidad Pu'blica de Navarra, 205-212.

Gruber, T.R., 1993. A translation approach to portable ontology specifications. Knowledge Acquisition, 5 (2), 199-221.

Kotis, K. and Vouros, G., 2006. Human-centered ontology engineering: the HCOME methodology. Knowledge and Information Systems, 10 (1), 109-131.

Kremer, R., 1994. Concept mapping: informal to formal [online]. In: W. Tepfenhart, J. Dick and J. Sowa, eds. Proceedings of the third international conference on conceptual structures, knowledge acquisition using conceptual graphs theory workshop, 19 August, University of Maryland College Park, MD, 152-167. Available from: http://sern.ucalgary.ca/ kremer/papers/ICCS94. html [Accessed 20 March 2010].

Lin, L.F., Lou, W.I., and Cai, M., 2011. Developing manufacturing ontologies for knowledge reuse in distributed manufacturing environment. International Journal of Production Research, 49 (2), 343-359.

Lo'pez, M., et al., 1999. Building a chemical ontology using methontology and the ontology design environment. IEEE Intelligent Systems, 14 (1), 37-46.

Maier, A., Schnurr, H.-P., and Sure, Y., 2003. Ontologybased information integration in the automotive industry. In: D. Fensel, K. Sycara, and J. Mylopoulos, J., eds. Proceedings of ISWC 2003, Lecture Notes in Computer Science, 2870 Berlin: Springer, 897-912.

Pereira, C., 2010. A organizac, ,ã o da informac,ã o e conhedmento em redes colaborativas como um processo de construc a o social do significado: uma teoria e um método prático. Thesis (PhD). Faculdade de Engenharia da Universidade do Porto, Porto, Portugal.

Pereira, C., Sousa, C., and Soares, A., 2009. A short-term semantic consensus: towards agile ontology specification for collaborative networks. In: IFIP advances in information and communication technology, leveraging knowledge for innovation in collaborative networks. Volume 307/ 2009. Boston: Springer, 301-310.

Pinto, S., et al., 2004. OntoEdit empowering SWAP: a case study in supporting DIstributed, Loosely-Controlled and evolvInG Engineering of oNTologies (DILIGENT). In: C.J. Bussler, et al., eds. The semantic web: research and applications. Berlin, Heidelberg: Springer, 16-30.
Pre'vot, L., et al., 2010. Ontology and the lexicon: a multidisciplinary perspective. In: H. Chu-Ren et al., eds. Ontology and the lexicon: a natural language processing perspective. Cambridge, UK: Cambridge University Press, 3-24.

Rajpathak, D. and Chougule, R., 2011. A generic ontology development framework for data integration and decision support in a distributed environment. International Journal of Computer Integrated Manufacturing, 24 (2), 154-170. doi:10.1080/0951192X.2010.531291.

Riss, U.V., et al., 2010. Knowledge work support by semantic task management. Computers in Industry, 61 (8), 798-805.

Roth, C. 2006. Co-evolution in epistemic networks reconstructing social complex systems. Structure and Dynamics: Journal of Anthropological and Related Sciences, 1(3), 5-160.

Ruikar, D., et al., 2007. Using the semantic web for project information management. Facilities, 25 (13/14), 507-524.

Soares, A., Azevedo, A., and de Sousa, J., 2000. Distributed planning and control systems for the virtual enterprise: organizational requirements and development life-cycle. Journal of Intelligent Manufacturing, 11, 253-270.

Staab, S., 2008. On understanding the collaborative construction of conceptualisations. In: International and interdisciplinary conference, processing text - technological resources, 13-15 March, Center for Interdisciplinary Research, Bielefeld University, Bielefeld, Germany.

Staab, S., et al., 2001. Knowledge processes and ontologies. IEEE Intelligent Systems, 16 (1), 26-34.

Sure, Y., 2002. A tool-supported methodology for ontologybased knowledge management. In: ISMIS 2002, 13th international symposium. Methodologies for Intelligent Systems, 27-29 June, Lyon, France.

Susskind, L., Mckearnan, S., and Thomas-Larmer, J., eds., 1999. The consensus building handbook: a comprehensive guide to reaching agreement. Thousand Oaks, CA: Sage Publications.

Ureten, S. and Ilter, H.K., 2006. Supply chain management ontology: towards an ontology-based SCM model. In: Third international conference on logistics and supply chain management [ILSCM 2006], 2-4 August, USA.

Vitasek, K., 2008. Supply chain management, terms and glossary [online]. The Council of Supply Chain Management Professional (CSCMP). Available from: http:// www.cscmp.org/Downloads/Resources/glossary03 [Accessed 16 November 2009].

Zhao, G., 2005. AKEM: an ontology engineering methodology. Deliverable 6.8 of FF POIROT project (Financial Fraud: Prevention Oriented Information Resources Using Ontology Technology) is an IST project funded by the European Commission). 